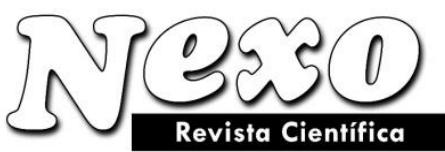

Vol. 32, No. 01, pp. 13-26/Junio 2019

\title{
PROPUESTA DE SEMILLEROS DE INVESTIGACIÓN PARA EL DESARROLLO DE LA COMPETENCIA INVESTIGADORA EN INGENIERÍAS
}

\section{PROPOSAL OF RESEARCH SEEDBEDS FOR THE DEVELOPMENT OF RESEARCH COMPETENCE IN ENGINEERING}

\author{
V. Guerrero Hernández ${ }^{1, *}$, A. Lagunes Domínguez ${ }^{2}$, C.A. Torres Gastelú ${ }^{3}$ J. Lau Noriega ${ }^{4}$ \\ ${ }^{1}$ Universidad Veracruzana, Facultad de Pedagogía, Boca del Río, Veracruz. México. \\ ${ }^{2}$ Universidad Veracruzana, Facultad de Contaduría y Administración, Ixtaczoquitlán, Veracruz. México. \\ ${ }^{3}$ Universidad Veracruzana, Facultad de Administración, Veracruz, Veracruz. México. \\ ${ }^{4}$ Universidad Veracruzana, Instituto de Ingeniería, Veracruz, Veracruz. México. \\ *zS13024361@estudiantes.uv.mx
}

(recibido/received: 23-Abril-2019; aceptado/accepted: 03-Junio-2019)

\begin{abstract}
RESUMEN
Este artículo trata sobre la importancia de la competencia investigadora en estudiantes universitarios que estudian alguna ingeniería y de una propuesta para su desarrollo. La metodología utilizada fue desarrollada en tres etapas, la primera etapa consistió en identificar la problemática de la falta de investigación en los países de América Latina, para este propósito se analizaron los rankings sobre investigación, los rankings de centros de investigación y el ranking de universidades, debido a que son los espacios en donde se debe de desarrollar la investigación en cada país. La segunda consistió en realizar el estado del arte sobre los esfuerzos realizados en cada país para alcanzar la competencia investigadora. La tercera etapa fue la descripción de la propuesta de semilleros de investigación para el desarrollo de dicha competencia. El resultado principal fue la propuesta de semilleros de investigación para el desarrollo de la competencia investigadora en ingenierías, la cual se está aplicando en este momento. La principal conclusión fue que el binomio de los semilleros de investigación y el aprendizaje combinado permitirán desarrollar la competencia investigadora.
\end{abstract}

Palabras claves: Semilleros; Competencias Investigativas; Ingenierías; Aprendizaje combinado.

\begin{abstract}
This paper deals with the importance of research competence in university students studying some engineering and a proposal for their development. The methodology used was developed in three phases, the first phase consisted of identifying the problem of lack of research in the countries of Latin America, for this purpose, research rankings, rankings of research centers and ranking of universities were analyzed, because they are the spaces where research should be developed in each country. The second phase consisted of making the state of the art about the efforts made in each country to achieve research competence. The third phase was the description of the proposal of research seedbeds for the development of said competence.
\end{abstract}




\section{Guerrero Hernández et al.}

The main result was the proposal of research seedbeds for the development of the research competence in engineering, which is being applied at this moment. In conclusion we have that the binomial of the research nurseries and the combined learning will allow to develop the research competence in engineering students.

Keywords: Seedbeds; Research competences; Engineering; Blended learning.

\section{INTRODUCCIÓN}

En la actualidad las Instituciones de Educación Superior (IES), en sus modelos educativos consideran importante la investigación y la innovación, ya que constituyen un eje integrador en el proceso de formación profesional.

Para Lagunes (2017) en su artículo el camino de la innovación educativa y su encuentro con las ingenierías, considera que la educación y las ingenierías han entrelazado sus caminos, existiendo cada día una conexión más fuerte. Los estudiantes de las ingenierías comúnmente resuelven problemas relacionados con problemáticas reales para el sector productivo, por lo tanto, las estrategias de los profesores es aplicar el aprendizaje basado en problemas. Lagunes (2017) propone dos estrategias de aprendizaje basado en proyectos para estudiantes de ingenierías, la primera es el Blended Learning y la segunda el Flipped Classroom.

Lagunes et al. (2015), en su trabajo de investigación sobre la multimodalidad como alternativa para el desarrollo de la competencia investigadora en estudiantes universitarios. La propuesta es la implementación de estrategias presenciales y virtuales que abonen al desarrollo de tan importante competencia.

Para Zamora (2014) en su artículo sobre la formación investigativa de los estudiantes: un problema aún por resolver, considera que el mundo de hoy requiere con urgencia que se encuentren respuestas viables al desarrollo de habilidades investigativas en los estudiantes, a partir de las formas de aprendizajes y los correspondientes métodos, técnicas, lo que supone que el docente desarrolle una enseñanza activa con el auxilio de métodos productivos con miras a cumplir con el encargo social que se plantea a la institución. En las siguientes líneas realizaremos una revisión de la literatura iniciando con el ámbito europeo, posteriormente América Latina y al final México.

Lazcano (2013), realizó un análisis descriptivo de la enseñanza sobre metodologías de investigación en los grados de periodismo en España, derivado de que, en revistas españolas especializadas en el área de comunicaciones, detectan una grave debilidad metodológica en los textos y resultados de sus investigaciones. Los resultados muestran la presencia de asignaturas sobre metodología de investigación en los grados de periodismo, pero solamente como un curso más, este estudio también determinó la ausencia de esta asignatura en siete universidades.

Rubio et al. (2016) realizaron un estudio para conocer la autopercepción de dominio que tienen los estudiantes de las competencias investigativas en el momento de la realización del trabajo de fin de grado de Pedagogía en la Universidad de Barcelona. Los resultados muestran niveles medios altos de percepción en general de las competencias investigativas, siendo mayor en escritura y técnicas de recogida y análisis cualitativo y menor en búsquedas bibliográficas especializadas, citación y técnicas cuantitativas. En cuanto a la investigación de los autores Rubio et al. (2016) se puede criticar que un estudio de autopercepción no comprueba nada, aunque aportan la percepción de los estudiantes.

Para Carrizo y González (2012), en su artículo sobre la importancia de la investigación en la formación de pregrado, reflexionan que la investigación en la universidad es un pilar fundamental para la generación del conocimiento y del desarrollo económico-social, considerando que la universidad en Cuba debe fomentar 


\section{Guerrero Hernández et al.}

el espíritu de investigación en todos los niveles de la formación académica, propiciando la formación integral de sus estudiantes de pregrado y posgrado, con la participación activa de sus docentes.

Estrada et al. (2014) en su artículo procedimiento para determinar las tendencias estadísticas del desarrollo de la competencia investigativas del Ingeniero en Ciencias Informáticas, su estudio fue cuantitativo cuyo objetivo fue desarrollar una aplicación para orientación de los tutores en la evaluación de la competencia investigativa de alumnos de la Universidad de Ciencias informáticas de Cuba. Con el uso de la aplicación se permite determinar la tendencia estadística del desarrollo de la competencia investigadora por cada uno de sus indicadores, además de conocer cuál será el desarrollo de cada indicador, en cada tarea, considerando sus evaluaciones históricas.

Morantes et al. (2017), en su publicación sobre la evaluación de un modelo didáctico integrador multimedia para la competencia investigadora, realizaron una investigación evaluativa con un diseño mixto. El propósito principal fue evaluar un modelo para el fomento de la competencia investigadora de los estudiantes de la escuela de eléctrica de la Universidad del Zulia Venezuela. Los resultados fueron favorables sobre el fomento de una cultura investigativa y trabajo en grupo desde el pregrado, mediante la concepción de una metodología de enseñanza y aprendizaje por investigación dirigida, estructurada en equipos cooperativos mediante un modelo didáctico integrador.

Rojas \& Méndez (2017), en su investigación evalúan la actitud estudiante a partir de los elementos de la autopercepción, incidencia de los profesores e incidencia de las universidades sobre la formación en investigación de ocho universidades del área metropolitana de Bucaramanga, Colombia. Los resultados muestran que la formación en investigación no se consolida a medida que el estudiante avanza en su trayectoria escolar. Sobre todo, no se consolida una cultura de la investigación ni interiorizar el valor social y la posibilidad de un futuro en la ciencia. Una baja actitud hacia la investigación por parte de los estudiantes de pregrado es una pésima señal para el presente y el futuro del desarrollo de la ciencia en el país. El problema es el desconocimiento de los alumnos como actores centrales del proceso de formación y de la falta de recursos pedagógicos para pretender un aprendizaje significativo.

Rojas \& Aguirre (2015), en su revisión bibliográfica identificaron los principales debates en investigaciones realizadas sobre la formación investigativa en la educación superior de América Latina. El análisis se llevó a cabo sobre tres aspectos, el primero fue la finalidad de la investigación formativa en cada contexto, la segunda es, de qué forma se están llevando a cabo los procesos de formación investigativa y, por último, cuáles son las condiciones institucionales desde las que se llevan a cabo dichos procesos. El hallazgo encontrado implica una articulación de las variables macro y micro que involucran las decisiones políticas para la inversión en ciencia y tecnología, así como aspectos asociados al currículo, prácticas pedagógicas y didácticas.

Para Díaz-Corrales et al. (2018), uno de los indicadores para la evaluación de la investigación y su impacto es el desarrollo de capacidades investigativas y de innovación. Además de considerar a la investigación científica como un medio de desarrollo profesional, tecnológico y económico de la sociedad y no solo un medio para la obtención del grado en la universidad.

López-De Parra, et al. (2017), realizaron un análisis al estado del arte sobre la producción investigativa en el ámbito internacional, durante los últimos cinco años (2010-2017) en América Latina. Uno de los temas que abordan es sobre los lugares de producción investigativa, los países que sobresalen son España, Cuba, Brasil, México, Venezuela y Colombia. España se destaca por su actividad significativa en el campo de la investigación y su preocupación versa sobre la formación de la misma. De igual manera en Latinoamérica; Colombia, Brasil, México y Venezuela abordan la problemática. Estados Unidos no se destaca en esta temática derivado de su gran avance científico, la enseñanza de la investigación no es considerada una problemática a indagar.

Nexo Revista Científica / Vol. 32, No. 01, pp. 13-26/Junio 2019 


\section{Guerrero Hernández et al.}

En México, la fundación de la Academia Mexicana de Ciencias (AMC) desde 1991 generó un programa de verano científico que tiene como propósito principal fomentar el interés de los jóvenes universitarios en cualquiera de sus áreas. Mediante la participación de una estancia de investigación de siete semanas en prestigiadas instituciones o centros de investigación del país, participando en proyectos que dan solución al sector productivo bajo la supervisión y asesoramiento de investigadores en activo, contribuyendo al logro de sus habilidades, conocimientos y aptitudes en investigación científica (AMC, 2019).

De igual manera cada año en la región centro del país, los estados de San Luis Potosí, Aguascalientes, Coahuila, Guanajuato, Querétaro y Zacatecas en conjunto con la Universidad Politécnica de San Luis Potosí, a través de la AMC convoca a la comunidad estudiantil a participar en el Verano de la Ciencia Región Centro. El objetivo principal de esta convocatoria es realizar una estancia con un periodo de cuatro semanas, donde profesores investigadores colaboran en un proyecto con estudiantes en actividades científicas.

Recientemente en el 2018, realizaron un estudio sobre veranos de la investigación-UG, alternativa de vinculación con el nivel superior y concluyen que el programa de verano es una estrategia de vinculación preliminar con el nivel superior de la UG, pues al ejecutar el método científico el alumno adquiere conocimientos, realiza una investigación y tiene actitudes que le permiten llevar a cabo una investigación, obtiene resultados que son presentados en un Congreso y adicionalmente son becados (Sandoval et al., 2018).

Roblero (2016), en su artículo desarrollo de la competencia investigativa en estudiantes de ciencias administrativas. El propósito principal del estudio fue describir el desarrollo de la competencia investigativa en estudiantes de ciencias administrativa de la Universidad Lindavista, Chiapas México. El estudio arrojó como resultados que la inclusión de asignaturas de investigación, la enseñanza centrada en el alumno y la exposición de éste a situaciones investigativas son percibidas como potenciadoras del desarrollo de la competencia investigadora.

\section{DESARROLLO}

Cuando se desea evaluar a un país para determinar que tanta investigación realiza, se pueden considerar diversos factores, en este caso para analizar la situación actual sobre investigación se analizan tres aspectos principales, el primero es el nivel de productividad en la divulgación del conocimiento por medio de artículos científicos, esto visto desde el ranking de investigación por país, el segundo es sobre el ranking mundial de los centros de investigación y el tercero es analizar el ranking de las universidades debido a que las universidades deben ser polos de investigación. A continuación, se detallan de dichos aspectos.

\subsection{Situación actual sobre el tema de investigación.}

En la actualidad la productividad en investigación la podemos medir con ranking a través SCImago Journal y Country Rank de las revistas y los indicadores científicos de los países desarrollados a partir de la información contenida en las bases de datos Scopus.

\subsubsection{Ranking en investigación}

Las revistas y los países se pueden comparar o analizar por separado, agrupados por 27 áreas temáticas, 313 categorías y 239 países en todo el mundo (SJR, 2017). A partir de la información obtenida por los indicadores, en la Tabla 1 se presentan los primeros diez países de la clasificación de América Latina, considerando su posicionamiento en el ranking del año 2017.

Nexo Revista Científica / Vol. 32, No. 01, pp. 13-26/Junio 2019 


\section{Guerrero Hernández et al.}

Tabla 1. Posición en el ranking mundial de los países de América Latina

\begin{tabular}{lllllllllllll}
\hline $\begin{array}{l}\text { N } \\
\text { o }\end{array}$ & País & 2017 & 2016 & 2015 & 2014 & 2013 & 2012 & 2011 & 2010 & 2009 & 2008 & 2007 \\
\hline 1 & Brasil & 14 & 14 & 13 & 13 & 13 & 13 & 13 & 13 & 14 & 14 & 14 \\
2 & México & 28 & 28 & 28 & 28 & 28 & 31 & 31 & 29 & 29 & 28 & 30 \\
3 & Argentina & 44 & 43 & 40 & 42 & 42 & 41 & 39 & 39 & 39 & 38 & 38 \\
4 & Chile & 45 & 44 & 44 & 44 & 46 & 46 & 46 & 45 & 44 & 43 & 43 \\
5 & Colombia & 47 & 48 & 47 & 47 & 48 & 49 & 52 & 52 & 53 & 52 & 53 \\
6 & Ecuador & 66 & 71 & 79 & 88 & 95 & 96 & 99 & 98 & 95 & 95 & 95 \\
7 & Perú & 73 & 72 & 72 & 76 & 77 & 75 & 72 & 73 & 73 & 75 & 74 \\
8 & Cuba & 79 & 77 & 69 & 68 & 64 & 64 & 64 & 65 & 59 & 59 & 58 \\
9 & Venezuela & 86 & 82 & 76 & 70 & 68 & 65 & 65 & 62 & 57 & 57 & 57 \\
10 & Uruguay & 88 & 83 & 86 & 80 & 84 & 83 & 78 & 79 & 76 & 76 & 77 \\
\hline \multicolumn{7}{r}{ Fuente: (SJR, 2017). }
\end{tabular}

El estado actual de la investigación en México muestra un reto respecto a la generación de ciencia e innovación aplicada, si nos comparamos con los tres primeros lugares del ranking mundial, Estados Unidos, China y Reino Unido. Ahora si se compara con los de la misma región, que son los países de América Latina, Brasil en el lugar 14, mientras que México está en el 29, Argentina en el 44, Chile en el 45 y Colombia en el 47, esto significa que México está muy lejos de alcanzar a Brasil (SJR, 2017).

México con datos de Scimago (2017), aparece en el número 28 a nivel mundial, posición que ha estado manteniendo desde el año 2013 después de que en 2012 hubiese estado en la posición 31.

En América Latina los cinco países con mayor presencia en investigación a nivel mundial son Brasil, México, Argentina, Chile y Colombia (ver Tabla 2). Se puede observar a México como uno de los primeros tres países latinos que da a conocer su aporte al conocimiento en diversas áreas, sin embargo, es importante observar que de acuerdo al indicador de cuartil (Q), respecto a la posición de una revista en relación con todas las de su área, se puede observar que en México las áreas que son mayormente reconocida es la revista de salud pública de México y la revista mexicana de astronomía y astrofísica.

Tabla 2. Comparativo de documentos científicos en los últimos 10 años en América Latina

\begin{tabular}{|c|c|c|c|c|c|c|c|c|c|c|c|}
\hline No & País & 2017 & 2016 & 2015 & 2014 & 2013 & 2012 & 2011 & 2010 & 2009 & 2008 \\
\hline 1 & Brasil & 73697 & 72174 & 68684 & 67293 & 63965 & 60393 & 54856 & 50402 & 46599 & 42116 \\
\hline 2 & México & 22954 & 22264 & 21018 & 21029 & 19547 & 18343 & 17106 & 15999 & 15092 & 14161 \\
\hline 3 & Argentina & 13308 & 13488 & 13347 & 13374 & 12248 & 12134 & 11628 & 10695 & 10076 & 8975 \\
\hline 4 & Chile & 12714 & 12930 & 11557 & 10999 & 9317 & 8907 & 7827 & 7113 & 6695 & 6034 \\
\hline 5 & Colombia & 10915 & 10141 & 8992 & 8317 & 7442 & 6585 & 5609 & 4910 & 4219 & 3630 \\
\hline 6 & Ecuador & 3172 & 2384 & 1657 & 1047 & 762 & 648 & 484 & 457 & 500 & 416 \\
\hline 7 & Perú & 2597 & 2354 & 2027 & 1721 & 1533 & 1374 & 1280 & 1085 & 971 & 799 \\
\hline 8 & Cuba & 1785 & 1970 & 2173 & 2309 & 2445 & 2379 & 2303 & 1996 & 2164 & 1887 \\
\hline 9 & Venezuela & 1530 & 1620 & 1780 & 2018 & 1972 & 2070 & 1975 & 2219 & 2400 & 2317 \\
\hline 10 & Uruguay & 1486 & 1560 & 1351 & 1453 & 1170 & 1105 & 1088 & 927 & 888 & 796 \\
\hline
\end{tabular}




\section{Guerrero Hernández et al.}

En un estudio realizado por López (2016) sobre la competitividad de la educación superior en cuatro países de América Latina, México muestra como fortaleza la capacidad de investigación y la administración de las universidades. Sin embargo, las instituciones de educación superior en México se mueven muy lejos de la competitividad mundial en investigación.

\subsubsection{Ranking de Centros de investigación}

El segundo aspecto a analizar en cuanto a investigación es el ranking mundial de centros de investigación, en este caso solo se tomará América Latina. En este ranking México tiene posicionado dentro de los primeros veinte lugares a 3 de sus centros de investigación, los cuales son el Centro de Investigación y de Estudios Avanzados del IPN CINVESTAV en la posición 113, el Instituto Nacional de Salud Pública en la posición 390 y con la posición 455 el Consejo Nacional de Ciencia y Tecnología CONACYT. Además, en la Tabla 3 podemos observar que Brasil es líder nuevamente, de los primeros 20 lugares de América Latina 13 son de Brasil, seguido por México y Argentina con 3 cada uno, y solo uno de Costa Rica (Webometrics, 2018).

Tabla 3. Ranking de Centros de Investigación en América Latina

\begin{tabular}{|c|c|c|c|}
\hline No & $\begin{array}{c}\text { Categoría } \\
\text { mundial }\end{array}$ & Centro de Investigación & País \\
\hline 1 & 89 & Fundacao Oswaldo Cruz & Brasil \\
\hline 2 & 108 & $\begin{array}{l}\text { Consejo Nacional de Investigaciones Científicas y } \\
\text { Técnicas }\end{array}$ & Argentina \\
\hline 3 & 113 & $\begin{array}{l}\text { Centro de Investigación y Estudios Avanzados del IPN } \\
\text { CINVESTAV }\end{array}$ & México \\
\hline 4 & 115 & Empresa Brasileira de Pesquisa Agropecuaria & Brasil \\
\hline 5 & 154 & Instituto Nacional de Pesquisas Espaciais & Brasil \\
\hline 6 & 185 & $\begin{array}{l}\text { Conselho Nacional de Desenvolvimento Científico e } \\
\text { Tecnológico }\end{array}$ & Brasil \\
\hline 7 & 350 & $\begin{array}{l}\text { Instituto Nacional de Tecnología Agropecuaria Buenos } \\
\text { Aires }\end{array}$ & Argentina \\
\hline 8 & 351 & Centro Brasileiro de Pesquisas Físicas & Brasil \\
\hline 9 & 390 & Instituto Nacional de Salud Pública & México \\
\hline 10 & 400 & Sociedade Beneficente Israelita Albert Einstein & Brasil \\
\hline 11 & 431 & Instituto Nacional de Pesquisas de Amazonia & Brasil \\
\hline 12 & 455 & Consejo Nacional de Ciencia y Tecnología CONACYT & México \\
\hline 13 & 456 & Instituto Nacional de Metrología & Brasil \\
\hline 14 & 466 & Instituto Nacional de Matemática Pura e Aplicada & Brasil \\
\hline 15 & 530 & Hospital Italiano de Buenos Aires & Argentina \\
\hline 16 & 649 & Instituto Brasileiro de Geografía e Estatística & Brasil \\
\hline 17 & 664 & Instituto Tecnológico de Aeronáutica & Brasil \\
\hline 18 & 701 & Observatorio Nacional & Brasil \\
\hline 19 & 733 & $\begin{array}{l}\text { Centro Agronómico tropical de Investigación y } \\
\text { Enseñanza }\end{array}$ & Costa Rica \\
\hline 20 & 734 & Laboratorio Nacional de Computación Científica & Brasil \\
\hline
\end{tabular}

\subsubsection{Ranking de Universidades}

El tercer aspecto a analizar son las universidades y como se encuentran en el ranking mundial, las tres universidades con mayor posicionamiento son la Universidad de Harvard, Universidad de Stanford y el Instituto Tecnológico de Massachusetts a nivel mundial. Desde luego México está muy alejado de tener una 


\section{Guerrero Hernández et al.}

universidad en los primeros lugares a nivel mundial, por ello se analizan solo las universidades de América Latina (ver Tabla 4).

Tabla 4. Ranking de universidades en América Latina

\begin{tabular}{llll}
\hline \multicolumn{1}{c}{ No } & \multicolumn{1}{c}{ Lugar } & \multicolumn{1}{c}{ Universidad } & \multicolumn{1}{c}{ País } \\
\hline 1 & 71 & Universidad de Sao Paulo (USP) & Brasil \\
2 & 121 & Universidad Autónoma de México (UNAM) & México \\
3 & 241 & Universidad Estadual de Campinas & Brasil \\
& & (UNICAMP) & \\
4 & 246 & Universidad Federal de Rio de Janeiro & Brasil \\
5 & 322 & Universidad de Chile & Chile \\
6 & 345 & Universidad Federal del Rio Grande del Sur & Brasil \\
& & (UFRGS & \\
7 & 372 & Universidad Eatadual Paulista Júlio de & Brasil \\
8 & 376 & Mesquita Filho & \\
9 & 422 & Universidad de Buenos Aires & Argentina \\
10 & 439 & Universidad Federal de Minas Gerais (UFSC) & Brasil \\
11 & 471 & Universidad Federal de Santa Catarina & Brasil \\
12 & 497 & Pontificia Universidad Católica de Chile & Chile \\
13 & 523 & Universidad Nacional de la Plata & Argentina \\
14 & 583 & Universidad Nacional de Colombia & Colombia \\
15 & 605 & Universidad de Puerto Rico & Brasil \\
16 & 606 & Universidad Federal de Paraná & Puerto Rico \\
& 608 & Universidad Federal Fluminense & Brasil \\
18 & 640 & Universidad de los Andes Colombia & Brasil \\
19 & 661 & CINVESTAV & Colombia \\
20 & 702 & Universidad Federal de Ceará & México \\
\hline Fuente: (Webometrics, 2018). & & Brasil \\
\hline
\end{tabular}

Los rankings dentro de su metodología ofrecen una fuerte comparación entre instituciones de educación superior a nivel mundial, respecto a diversos indicadores. Tomando en cuenta el conteo de Julio 2018 de acuerdo a Ranking Web de Universidades, dentro de las primeras veinte universidades latinas, el primer lugar se lo llevo la Universidad de Sau Paulo en Brasil y el segundo lugar se encuentra la Universidad Autónoma de México, considerando que ocupa el lugar 141 a nivel mundial (Webometrics, 2018).

Se observa que 11 universidades brasileñas están dentro de las veinte mejores universidades, muy lejos de Brasil, Argentina, Chile, Colombia y México cada país con 2 universidades y al final Puerto Rico con 1 dentro de este ranking (Webometrics, 2018). Por lo que se refiere a México de las diez mejores universidades, el estado de Veracruz que es el estado en donde se realiza la presente investigación, ocupa el lugar 74 solamente con la Universidad Veracruzana (Webometrics, 2018).

En México se cuenta con una gran diversidad de instituciones de educación superior, los Institutos Tecnológicos de México, constituyen el Tecnológico Nacional de México (TecNM), un grupo de instituciones de educación superior pública de la República Mexicana, las cuales ofrecen programas educativos para ingenierías. El TecNM se encuentra dividido por 266 Instituciones, 126 Federales y 134 descentralizadas llamadas Institutos Tecnológicos Superiores (Webometrics, 2018). 


\section{Guerrero Hernández et al.}

\subsection{Posibles causas}

Una vez demostrado y reconocido el problema de la investigación en América Latina y en México, las posibles causas es no estar invirtiendo en ciencia y tecnología o lo que se invierte es muy poco que no alcanza a hacer diferencia. Por ejemplo, la organización para la cooperación y el desarrollo económico (OCDE), en el 2015 realizó diversas estadísticas, dentro de las cuales existe una comparación entre países iberoamericanos asociados a ella sobre indicadores en ciencia y tecnología.

Un indicador corresponde al gasto interno bruto per cápita en investigación y desarrollo de la población per cápita, la estadística muestra un total de 41 países, apareciendo México, con el 98.4 per cápita a diferencia de Estados Unidos con el 1,563.2 per cápita y de Israel con el 1,556 per cápita que es el más alto en el comparativo. Esto significa que el gasto interno bruto per cápita en investigación de México es muy bajo (De Moya, 2018).

Una segunda causa es el porcentaje del gasto interno bruto en investigación y su desarrollo de la población, México cuenta con el $0.6 \%$, Estados Unidos con el 2.8\% comparados contra Israel con el $4.3 \%$ del gasto interno bruto. Nuevamente México muy por debajo de lo deseado en cuanto a su inversión en investigación. Se puede mencionar otro ejemplo, el SIR World 2018 que muestra la inversión en I+D de los países de América Latina representa el 3,4\% de la inversión mundial y sus investigadores el $4,1 \%$ de los recursos humanos dedicados a investigación. Esto se refleja en el comportamiento de la región que a pesar de que consigue aumentar su presencia en el ranking en 51 instituciones, continúa estando en general muy lejos de las posiciones destacadas en el ranking mundial.

Otro factor importante en México es la participación del personal de investigación y desarrollo en la empresa es del $1 \%$, se encuentra entre los más bajos de la OCDE y es considerablemente menor que en países como Israel, Finlandia y Dinamarca, donde superan el 20\% (OCDE, 2017).

\subsection{Pronóstico sobre posibles situaciones}

Considerando la investigación realizada por Sánchez-Vidal et al. (2018) sobre expectativa del desarrollo de la investigación en México en el marco de Latinoamérica, los autores realizan una proyección del desempeño en la investigación de los países de América Latina, basándose en los indicadores SJR 2017 mediante un método del incremento porcentual relativo, también consideraron la proyección con una tasa constante permitiendo identificar un pronóstico de posibles situaciones.

Realizaron una proyección incremental a 40 años de los primeros 7 países en la clasificación de Latinoamérica en el ranking de investigación con base a los documentos publicados previamente.

Tabla 5. Proyección incremental a 40 años de los primeros 7 países en la clasificación de Latinoamérica.

\begin{tabular}{cccccc}
\hline $\begin{array}{c}\text { Posición } \\
2016\end{array}$ & País & $\begin{array}{c}\text { A 12 años } \\
\text { (3er lugar) }\end{array}$ & $\begin{array}{c}\text { A 23 años } \\
\left(4^{\circ} \text { lugar }\right)\end{array}$ & $\begin{array}{c}\text { A 24 años } \\
\left(5^{\circ} \text { lugar }\right)\end{array}$ & $\begin{array}{c}\text { A 40 años } \\
\left(6^{\circ} \text { lugar }\right)\end{array}$ \\
\hline 1 & Brasil & $241,596.84$ & $762,976.32$ & $847,057.43$ & $4,511,715.09$ \\
2 & México & $52,840.77$ & $123,091.60$ & $132,927.75$ & $454,790.04$ \\
3 & Argentina & $25,856.88$ & $49,035.38$ & $51,972.81$ & $131,838.74$ \\
4 & Chile & $41,416.87$ & $124,666.15$ & $137,801.84$ & $684,476.66$ \\
5 & Colombia & $54,095.57$ & $266,958.39$ & $308,652.08$ & $3,146,873.16$ \\
6 & Ecuador & $17,419.85$ & $113,765.73$ & $134,927.16$ & $2,067,787.88$ \\
7 & Perú & $11,033.52$ & $47,820.40$ & $54,640.31$ & $461,223.83$ \\
\hline Fuente: Sánchez, A., et al., 2018 & & & &
\end{tabular}




\section{Guerrero Hernández et al.}

Como se observa en la Tabla 5, en esta proyección México inicia en la posición 2 del ranking de América Latina, sería superado por Colombia a los 12 años, por Chile a los 23 años, por Ecuador a los 24 años y por Perú a los 40 años, esto es, pasaría del segundo lugar al sexto en 40 años, lo cual mediante la técnica aplicada por los autores auguran un futuro decadente para la investigación en México.

México presenta inminentemente una expectativa de desarrollo poco alentadora, mientras que para otros países de la región su expectativa es de buena a muy buena como Colombia, Chile, Ecuador y Perú. A tal grado que, en las dos proyecciones desarrolladas por Sánchez-Vidal et al. (2018) se estima que México será desplazado de las primeras posiciones en América Latina.

En cuanto a los centros de investigación y a las universidades en América Latina, actualmente se muestra que hay una clara tendencia negativa en el posicionamiento de México, donde Brasil cada vez se vuelve más fuerte. Para mejorar este panorama, existen diversas alternativas que pueden ayudar a cambiar esta tendencia.

\subsection{Propuesta de solución}

Una manera de control al pronóstico es una solución para superar la situación actual, la propuesta que se da son los semilleros de investigación para el desarrollo de la competencia investigadora en ingenierías. Un alumno universitario en la actualidad debe tener las habilidades, conocimientos y actitudes necesarias para que durante su vida estudiantil y profesional sean competentes en investigación. Considerando que no necesariamente vaya a ser un científico, pero sí que pueda desempeñarse profesionalmente según las necesidades locales sin perder la visión global de lo que pueda estar sucediendo en el mundo, además de fortalecer sus facultades individuales en la interacción con otros investigadores o profesionistas. El alumno debe generar proyectos de investigación innovadores que den solución a problemas reales de su ámbito de origen, atendiendo a necesidades locales y generando un intercambio con la sociedad con nuevos hallazgos y conocimiento.

2.5 Propuesta de semilleros de investigación para el desarrollo de la competencia investigadora en ingenierías

La propuesta de semilleros de investigación para el desarrollo de la competencia investigadora en ingenierías se basó en dos experiencias, la primera son los semilleros de investigación, espacios extracurriculares que utilizan las universidades colombianas, para Abello y Baeza (2007) explican el caso de la Universidad del Norte en Colombia y los diez años de experiencia de implementación de un programa de semilleros de investigación como estrategia de formación investigativa, los resultados que obtuvieron son de 397 jóvenes formados en investigación. Existe una gran diferencia del 2003 y 2006 respecto a la obtención de grados con reconocimiento de excelencia de los trabajos de tesis, así como la visibilidad de la investigación estudiantil respecto a concursos nacionales y premios. La universidad del Norte ha sido ganadora de los tres primeros lugares en el área de tecnologías apropiadas y de desarrollo sostenible y medio ambiente.

Es importante analizar algunas experiencias sobre semilleros de investigación como alternativa y ya que casi no son implementados en México. Saavedra et al. (2015) en su análisis sobre semilleros de investigación, concluye que son escenarios alternativos de discusión académica e investigación que permiten fortalecer las habilidades investigativas de los estudiantes. Los semilleros de investigación se consolidan en Colombia como una herramienta capaz de generar los cimientos investigativos para futuros profesionales que necesitan las naciones. De acuerdo con Patiño (2007) los semilleros de investigación permiten la generación de nuevos modelos pedagógicos de interacción de conocimientos entre estudiantes y docentes. Lo anterior hace reflexionar que en la actualidad no existe en las universidades mexicanas la cultura de trabajo colaborativo entre docentes y estudiantes que permita dar solución a proyectos reales que 
formen parte de las líneas de investigación y cuerpos académicos, permitiendo formarse en las habilidades, conocimientos y aptitudes en investigación (ver Figura 1).

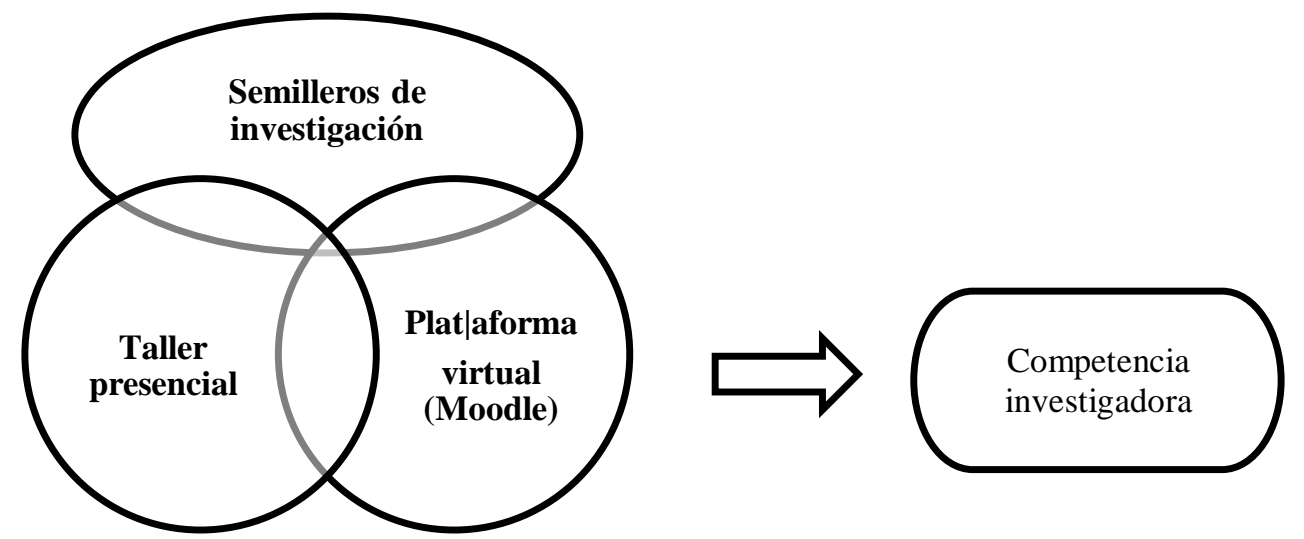

Figura 1. Propuesta de semilleros de investigación para el desarrollo de la competencia investigadora en ingenierías. Fuente: Elaboración propia

\subsubsection{Diseño y desarrollo de los semilleros de investigación.}

Los semilleros de investigación son espacios extracurriculares en donde un grupo de estudiantes desarrollan la competencia investigadora, apoyados por un docente que cumpla el rol de orientador teórico y metodológico. Partiendo de que el trabajo de investigación busca determinar si un semillero de investigación permite incrementar la competencia investigadora en estudiantes universitarios, se diseñó y desarrollo el reglamento para los semilleros de investigación que consta de ocho apartados, (1) objetivos; (2) estructura y conformación; (3) de los requisitos; (4) de las funciones del líder estudiantil y del comité del semillero; (5) de los incentivos; (6) de los derechos de autor; (7) del retiro de miembros o disolución del semillero de investigación; y (8) de los beneficios.

La conformación de los semilleros de investigación fue con estudiantes que pertenecen a un Instituto de educación superior adscrita a Tecnológico Nacional de México (TecNM), en consecuencia, se revisó su normatividad para considerar lo establecido en el tema de investigación en su modelo educativo para el siglo XXI: Formación y desarrollo de competencias profesionales, principalmente la participación de los estudiantes. Además de considerar uno de los seis ejes que darán dirección y articulación a la propuesta del nuevo modelo educativo del TecNM 2018; el eje 3 Investigación, Innovación y emprendimiento para el fomento y difusión de la cultura en investigación.

Otro aspecto importante que se considera, son los momentos del proceso integrador de titulación, específicamente el primer momento, al cursar la asignatura de fundamentos de investigación. De acuerdo a lo anterior, la conformación de los semilleros de investigación se lleva a cabo de la siguiente manera:

- El estudiante cursa y aprueba la asignatura de Fundamentos de Investigación.

- Publicación de la convocatoria e invitación a formar parte de los semilleros de investigación a los estudiantes de fundamentos de investigación, vía correo electrónico, personalmente y mediante carteles.

- Los estudiantes interesados en formar parte de los semilleros de investigación como trabajo extracurricular solicitan registrarse ante la coordinación de semilleros.

- Los Cuerpos Académicos (CA) presentan a los estudiantes las líneas de investigación y proyectos de investigación vigentes.

- Se aplica a los estudiantes una evaluación diagnóstica (preprueba) de la competencia investigadora. 


\section{Guerrero Hernández et al.}

- El estudiante ingresa a una formación sobre las cinco categorías que integran a la competencia investigadora.

- Los estudiantes son asignados a un docente de un cuerpo académico o líder de proyecto de investigación de acuerdo a sus resultados de la evaluación, el docente fungirá de tutor durante su estancia en los semilleros, además de asegurar de que el objeto de estudio sea abordado de manera original.

- El semillero de investigación está integrado por tres alumnos y un docente como mínimo.

- El estudiante trabaja en los semilleros de investigación hasta terminar de cursar la asignatura de Taller de Investigación II, entregando un reporte final, momento en el que inicia su proceso integrador de titulación, con la entrega de un proyecto de investigación.

- El estudiante y docente asignado deben generar producción académica derivados de los proyectos de investigación que estén desarrollando.

- Se aplica a los estudiantes una evaluación final (posprueba) de la competencia investigadora para valorar su continuidad en los momentos siguientes del proceso integrador de titulación.

Posteriormente, se realizó la planeación y programación del programa de formación de la competencia investigadora en modalidad de aprendizaje combinado por medio de actividades presenciales con talleres y las actividades virtuales en la plataforma Moodle. Las planeaciones de las actividades presenciales constaron de talleres que se organizaron en cinco sesiones presenciales impartidos en dos periodos, el primero fue al inicio del ciclo escolar enero - junio 2018, los temas que se abordaron por taller fueron sobre, (1) conceptos sobre investigación; (2) búsquedas avanzadas y Google académico; (3) planeación de la investigación. El segundo periodo fue en el mes de abril, del mismo ciclo escolar, los temas que se abordaron fueron sobre, (4) procesamiento y análisis de la información; y (5) aparato crítico y formato APA $6^{a}$ edición. Esta formación tuvo una duración de 20 hrs. en cinco sesiones de 4 hrs. cada una incluyendo exposición oral, ejercicios de reforzamiento, retroalimentación y soporte académico por parte de su docente tutor y el facilitador de los talleres. El 100\% de los sujetos completó esta formación de manera satisfactoria.

En las planeaciones de las actividades virtuales, se proporcionó el sitio e instrucciones para realizar las actividades virtuales en la plataforma de Moodle, sitio donde se alojaron los recursos complementarios de las actividades presenciales de cada uno de los cinco talleres antes expuestos. En la plataforma se generó un curso llamado semilleros de investigación, consta de cinco módulos que llevan por nombre, (Módulo 1) conceptos sobre investigación; (Módulo 2) búsquedas avanzadas y Google académico; (Módulo 3) planeación de la investigación; (Módulo 4) procesamiento y análisis de la información; y (Módulo 5) aparato crítico y formato APA $6^{a}$ edición. Cada módulo se conformó de cuatro secciones, en la primera sección se daba contexto sobre lo que se abordaba en cada módulo. En la segunda sección se colocaron los materiales proporcionados en cada taller presencial, haciendo uso del recurso archivo de Moodle que permite proveer de un archivo como recurso del curso. En la tercera sección del módulo corresponde a las actividades, se utilizó el recurso tareas de Moodle que permite evaluar el aprendizaje de los estudiantes mediante la creación de una tarea, el recurso tarea fue colocado en cada módulo para la entrega de las evidencias en la conformación de su protocolo de investigación como producto final de la formación.

Finalmente, la cuarta sección corresponde a las preguntas frecuentes, se utiliza el recurso foro de Moodle que permite a los participantes tener discusiones asincrónicas, se implementó en cada módulo un foro que permitiera iniciar una discusión con los temas que surgieran como dudas individuales o grupales permitiendo interactuar entre los estudiantes para colaborar en las respuestas de manera oportuna junto con el facilitador. Estas actividades tuvieron una duración de $40 \mathrm{hrs}$. totalmente virtual solo respetando las fechas de entrega de cada evidencia. El 100\% de los sujetos completó esta formación de manera satisfactoria. En la figura 1 se puede observar la propuesta de semilleros de investigación para el desarrollo de la competencia investigadora en ingenierías. 


\section{Guerrero Hernández et al.}

\section{CONCLUSIONES}

Se puede decir que este trabajo de investigación nos proporciona dos conclusiones y una línea de investigación. En cuanto a la primera conclusión, podemos afirmar que la investigación es un tema de suma importancia para América Latina pero además es un tema pendiente de mejorar por sus bajos niveles comparados con otras regiones del mundo.

La segunda conclusión es que México debe invertir más en investigación y no solo eso, sino que invertir de mejor manera en esta competencia si no quiere bajar la producción de documentos científicos y por ende en los ranking mundiales.

Finalmente, como una línea de investigación futura, México debe apostar a los semilleros de investigación para el desarrollo de la competencia investigadora en ingenierías, ya que es una manera de aprovechar a los profesores y su experiencia, aprovechar las tecnologías y sus bondades, y sobre todo, aprovechar a los estudiantes y su energía.

\section{REFERENCIAS}

Abello, R. \& Dáger, B. (2007). Estrategia de información investigativa en jóvenes universitarios: caso Universidad del Norte, Studiositas, 2 (2), 43-56.

AMC (23 de enero 2019). Academia Mexicana de Ciencias. Recuperado de https://www.amc.edu.mx/amc/index.php?option=com content $\&$ view=article\&id=59\&Itemid=79

Carrizo, J. \& González, M. (2012). Importancia de la investigación en la formación de pregrado. Congreso Universidad, 2(2), ISSN 2306-918X. Recuperado de http://www.congresouniversidad.cu/revista/index.php/rcu/article/view/348

De Moya, F., Herrán, E., Bustos, A., Corera, E. \& Tibaná, G. (2018). Ranking Iberoamericano de instituciones de educación superior. SIR Iber 2018. Barcelona, España: Ediciones Profesionales de la Información SL. ISBN:978 840903911 1, https://doi.org/10.3145/sir-iber-2018.

Díaz-Corrales, A. et al. (2018). Metodologías e indicadores académicos, económicos, sociales y tecnológicos para la evaluación del impacto de la investigación científica universitaria, Nexo Revista Científica. 31(02), 74-88.

Estrada-Molina, O et al. (2014). Procedimiento para determinar las tendencias estadísticas del desarrollo de la competencia investigativas del Ingeniero en Ciencias Informáticas, Gecontec: Revista Internacional de Gestión del Conocimiento y la Tecnología, 2(2), Recuperado de https://ssrn.com/abstract=2438032

Lagunes, A. (2017). El camino de la innovación educativa y su encuentro con las ingenierías. Ingenierías USBMed, 8(1), 4-6.

Lagunes, A. (2016). Development Of A Research Competence In University Students Through Blended Learning, Tojet: The Turkish Online Journal of Educational Technology, 668-673.

Lagunes, A., Flores, M.A., Torres, C. A. \& Castro (2015). La competencia investigadora en los universitarios mediante el empleo didáctico de los materiales multimedia. Trabajo presentado en el X Congreso Internacional sobre el enfoque basado en competencias, At Medellin, Colombia. Resumen recuperado 


\section{Guerrero Hernández et al.}

https://www.researchgate.net/publication/271197480_La_competencia_investigadora_en_los_universitari os mediante el empleo didactico de los materiales multimedia.

Lazcano, D. (2013), Metodologías de investigación: aproximación a su enseñanza en el grado de periodismo en España, Periodística, 15, 11-28. Recuperado de http://www.publicacions.iec.cat / DOI: 10.2436/20.3008.02.26 ISSN

López, L., Polanco, V., \& Correa, L. (2017). Mirada de las investigaciones sobre formación investigativa en la universidad latinoamericana: estado del arte (2010-2017). Rev.investig.desarro.innov, 8(1), 77-95. DOI: 10.19053/20278306.v8.n1.2017.7371

Morantes, Z. et al. (2017). Evaluación de un modelo didáctico integrador multimedia para la competencia investigadora, Telos, 19(3), 450-474.

OCDE (2017). Diagnóstico de la OCDE sobre la estrategia de competencias, destrezas y habilidades en México, Ediciones OCDE. Recuperado de https://www.oecd.org/mexico/Diagnostico-de-la-OCDE-sobre$\underline{\text { la-Estrategia-de-Competencias-Destrezas-y-Habilidades-de-Mexico-Resumen-Ejecutivo.pdf }}$

Rojas, C. \& Aguirre, S. (2015). La formación investigativa en la educación superior en América Latina y el Caribe: una aproximación a su estado del arte. Revista Eleuthera, 12, 197-222. 10.17151/eleu.2015.12.11.

Rojas, M. \& Méndez, R. (2017). Procesos de formación en investigación en la Universidad: ¿Qué les queda a los estudiantes? Sophia, 13(2), 53-69.

Roblero, G. (2016). Desarrollo de la competencia investigativa en estudiantes de ciencias administrativas, Coloquio de Investigación de ANFECA región sureste. Recuperado de https://www.researchgate.net/profile/Gudiel Roblero/publication/301200180 Desarrollo de Competenci as Investigativas en Estudiantes de Ciencias Administrativas/links/570bc1d808ae8883a1ffd771.pdf

Rubio et al. (2016). Autopercepción de las competencias investigativas en estudiantes de último curso de Pedagogía en la Universidad de Barcelona para desarrollar su trabajo de fin de grado. Revista Complutense de Educación, 29(2), 335-354.

Saavedra-Cantor, C. et al. (2015). Semilleros de investigación: desarrollos y desafíos para la formación en pregrado, Educ. Educ., 18(3), 391-407, DOI: 10.5294/edu.2015.18.3.2

Sánchez, A. et al. (2018). Expectativa del desarrollo de la investigación en México en el marco de Latinoamérica, Memorias del Congreso Internacional EduAction Miami 2018, Primera edición ISBN: 978 0-9915776-7-5, 248-264

Sandoval, M. et al. (2018). Veranos de la Investigación UG alternativa de vinculación con el nivel superior, Congresos CLABES. Recuperado de http://revistas.utp.ac.pa/index.php/clabes/article/view/1959

SJR (24 de enero 2019). Scimago Journal \& Country Rank. Recuperado de http://www.scimagojr.com. Webometrics (18 de noviembre 2018). Ranking Web de Centros de Investigación. Recuperado de http://research.webometrics.info/es/Latin_America es

Webometrics (28 de noviembre 2018). Ranking Web de Universidades. Recuperado de http://www.webometrics.info/es/Latin America es.

Zamora, N. (2014). La formación investigativa de los estudiantes: un problema aún por resolver. Escenarios, 12(2), 76-85. 


\section{Guerrero Hernández et al.}

\section{SEMBLANZA DE LOS AUTORES}

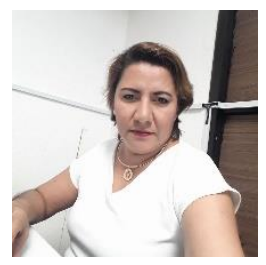

Verónica Guerrero-Hernández: Maestría en Tecnologías de Información por la Universidad Cristóbal Colon de Veracruz, Ver. México y Licenciada en Informática por el Instituto Tecnológico de Orizaba, México. Profesora de tiempo completo en el Instituto Tecnológico Superior de San Andrés Tuxtla desde 1997. Reconocimiento de Perfil Deseable PRODEP del 2013 - 2016. Trabaja en líneas de investigación vinculadas con el desarrollo de tecnologías emergentes

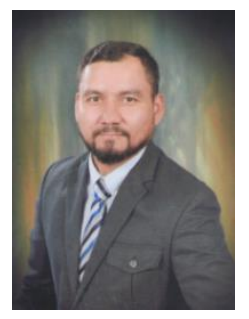

Agustin Lagunes-Domínguez: Doctor en Sistemas y Ambientes Educativos por la Universidad Autónoma de Madrid con mención Cum Laude. Arbitro en revistas Scopus, revistas en el padrón de CONACYT y en revistas indexadas de Ecuador, Colombia, México y otros países. Estancias en la Universidad de Magallanes en la Patagonia Chilena, en Southern University en Louisiana, Estados Unidos, en la Universidad Autónoma de Madrid, en la Universidad San Buenaventura en Medellín, en el Politécnico Colombiano Jaime Isaza Cadavid y en la Universidad Nacional Mayor de San Marcos. Reconocimiento de Perfil Deseable PROMEP del 2008 a la fecha. Par evaluador del Comité Interinstitucional para la Evaluación de la Educación Superior (CIEES). Integrante del Consejo Editorial de la Universidad Veracruzana. Profesor de tiempo completo de la Universidad Veracruzana desde el año 2000. Profesor del Doctorado en Sistemas y Ambientes Educativos reconocido en el Programa Nacional de Posgrado de Calidad (PNPC). Coordinador general de "Red de Investigación Multidisciplinar para la Cultura Investigadora" (RIMCI). Candidato en el Sistema Nacional de Investigadores (SNI).

Carlos Arturo Torres-Gastelú: Doctor en Ciencias de la Administración, maestro en Ciencias de

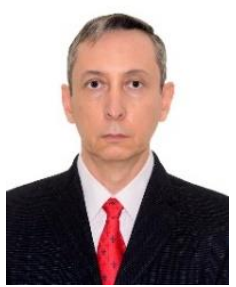
la Computación y Licenciado en Informática. Obtuvo mención honorífica a nivel de doctorado y maestría, así como titulación automática en el nivel de licenciatura. Recibió la medalla de plata Alfonso Caso por haber sido el graduado más distinguido en 2006 del programa de doctorado en Ciencias de la Administración en la UNAM. Ejerció en la industria durante 13 años (1988 a 2001) en el sector de servicios de informática. Desde hace 20 años se desempeña como académico de tiempo completo en la Universidad Veracruzana. Posee el reconocimiento de profesor con Perfil Deseable PRODEP desde 2008 hasta la fecha. Forma parte del Sistema Nacional de Investigadores del CONACYT en el nivel 1 (2015-2021). Tiene 175 publicaciones (6 libros, 34 capítulos de libro, 49 artículos científicos, 86 artículos en extenso). Su línea de investigación es el aprendizaje mediado por las TIC.

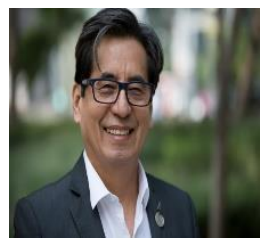

Jesús Lau-Noriega: Investigador del Instituto de Ingeniería, y profesor del Programa AFEL y del Doctorado en Sistemas y Ambientes Educativos de la Facultad de Peagogía, Universidad Veracruzana, Región Veracruz, y miembro ininterrumpido del Sistema Nacional de Investigadores desde 1989 (29 años). Doctorado (Ph.D) en ciencias de la información (Universidad de Sheffield, Inglaterra); maestría en bibliotecología (Universidad de Denver, Colorado, EUA); y licenciatura en derecho (Universidad Autónoma de Sinaloa). Con más de 200 ponencias y artículos de revistas y así como 20 libros editados en el campo informativo. El Dr. Lau ha recibido distintos reconocimientos en México, Estados Unidos, Cuba y Francia. Ha viajado a cerca de 70 países por motivos profesionales.

Nexo Revista Científica / Vol. 32, No. 01, pp. 13-26/Junio 2019 